\title{
MAGNETOPHOTOCONDUCTIVITY DUE TO INTRA-SHALLOW-DONOR TRANSITIONS IN SEMI-INSULATING GaAs*
}

\author{
K. KARPIERz ${ }^{a}$, M.L. SADOWSKI ${ }^{b}$ AND M. GRYNBerg ${ }^{a}$ \\ ${ }^{a}$ Institute of Experimental Physics, Warsaw University \\ Hoża 69, 00-681 Warszawa, Poland \\ ${ }^{b}$ High Pressure Research Centre UNIPRESS, Polish Academy of Sciences \\ Sokolowska 29/37, 01-142 Warszawa, Poland
}

\begin{abstract}
The paper concerns investigations of shallow centres in semi-insulating (SI) GaAs samples. A very sensitive measuring method - magnetophotoconductivity due to intra-shallow-donor transitions - was used. We report the behaviour of intra-impurity transitions as well as an additional structure in low magnetic fields for different far-infrared wavelengths $(70.6 \mu \mathrm{m}, 96.5 \mu \mathrm{m}$, $118.8 \mu \mathrm{m}, 163 \mu \mathrm{m})$. The results are discussed in terms of a fluctuating potential from ionized centres in SI GaAs. The physical mechanism responsible for the low magnetic field structure is proposed.
\end{abstract}

PACS numbers: 72.40+w, 78.20.Ls, 78.50.-w

Far-infrared (FIR) magnetophotoconductivity is a well-known method [1, 2] of investigating shallow states in semiconductors. Its application to SI GaAs has been reported previously [3]. The case of SI GaAs is more complicated than that of e.g. $n$-type GaAs. Due to the pinning of the Fermi level by the midgap EL2 defect there are no electrons in the conduction band and all shallow states are unoccupied by electrons. Applying external illumination (in our experiments a constant flux of photons with $\lambda=0.86 \mu \mathrm{m}$ ) it is possible to obtain $[3,4]$ a sufficiently numerous population of neutral shallow donors to investigate them. The samplet is then additionally illuminated with FIR light in order to obtain intra-shallow-donor transitions. The increase of photoconductivity due to chopped FIR light is measured using a lock-in amplifier. Typical spectra for different wavelengths are presented in Fig. 1(a). The well-pronounced peaks correspond to the

*This work was supported in part by G-MEN 131/90.

tThe sample was a rectangular slab $(3.0 \times 1.0 \times 0.5 \mathrm{~mm})$ of bulk SI GaAs (grown by the LEC technique in the (100) direction) with a concentration of the EL2 defect about $10^{16} \mathrm{~cm}^{-3}$. The dark resistance at $4.2 \mathrm{~K}$ was of the order of $10^{12} \Omega$. Two nonrectifying (down to $4.2 \mathrm{~K}$ ) Au-Ge-Ni contacts were alloyed by dr E. Kamińska and dr A. Piotrowska from the Institute of Electron Technology in Warsaw. 


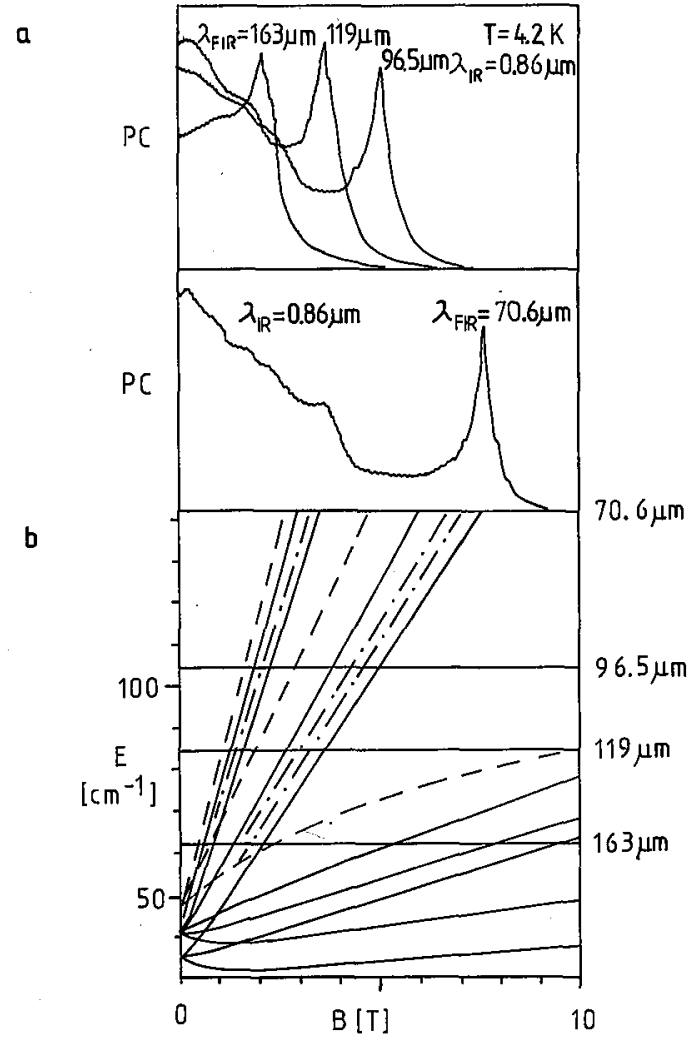

Fig. 1. (a) Photoconductivity of SI GaAs due to FIR illumination for different FIR wavelengths versus magnetic field. Vertical scales of all spectra were normalized. $\lambda_{\mathrm{IR}}$ and $\lambda_{\text {FIR }}$ signify the wavelengths of the incident infrared and far infrared light, respectively. (b) Magnetic field dependences of the energy differences between the $1 \mathrm{~s}$ state and excited states of a shallow dơnor in GaAs. Broken lines correspond to 1s-LL energy differences (after [1]).

$1 s-2 p_{+}$transition in the hydrogen-like shallow donor system (compare with Fig. $1(\mathrm{~b}))$. In contrast to $n$-GaAs there is a broad structure visible at low magnetic fields (also reported previously [3]). A broad structure at magnetic field lower than the $1 s-2 p_{+}$transition was also observed by Paget and Klein [5] in an absorption experiment and was attributed to transitions in shallow donors with greater binding energies. Our results differ from theirs and do not confirm this hypothesis. In Fig. 1(a) one can see for all presented FIR wavelengths a broad bump centred at about zero magnetic field which vanishes gradually with $B$. Additionally, for $B$ greater than that at which the $1 s-2 p_{+}$transition occurs the photoconductive signal decreases rapidly and then disappears. Analyzing this result we see that sweeping the magnetic field we make a scan along a horizontal line on Fig. 1(b). Starting from $0 \mathrm{~T}$ we meet an infinite number of excited shallow donor states and 
Landau Levels LL (not all are plotted). When $B$ is increased past the $1 s-2 p_{+}$transition there are no more states except the continuum of the LL with $n=0$. The direct photoionization of electrons from the $1 s$ state into the continuum of $n=0$ LL is very weak and practically not observable. This is not due to small density of states for LL because with increasing magnetic field (and density of states) the photoconductive signal decreases. Considering the physical mechanism of photoconductivity one should remember that in this system it is quite complicated. It consists of at least two steps. First: the absorbed FIR photon transfers an electron from the ground state to an excited state. Second: the electron is transferred from the excited state into the conduction band [6]. The excited states in this case are resonant with the continuum of LL (at least with $n=0 \mathrm{LL}-$ see Fig. 1(b)). The registered photocurrent $P C$ is proportional to the product of probabilities of both processes:

$$
P C \sim\langle\text { exc. state }|\hat{p}| \text { ground state }\rangle\langle\mathrm{LL}|\hat{m}| \text { exc. state }\rangle,
$$

where $\hat{m}$ is an operator describing transfer mechanism which will not be discussed. The first term in the above equation is a matrix element of the transition in a hydrogen atom in a magnetic field. The second term describes the probability of the autoionization of a resonant excited state. In our experiments both probabilities should be determined for the case of intermediate magnetic fields. Such a theoretical, numerical calculation has been done [7] and its authors conclude that there are two kinds of excited states of a hydrogen atom in an intermediate magnetic field. The first are "ordinary" states which correspond to those obtained in the limit of low magnetic fields. The second kind are states which perform radiationless transitions to the continuum of one of the lower-lying LL. They do not correspond to states in the limit of weak magnetic fields. In Fig. 1(b) they are marked as dashed-dotted lines. The above presented considerations are valid both for $n$-type and SI GaAs. However, in the SI material one deals with strong local electric fields [3] due to numerous ionized impurities and total lack of free electrons and therefore weak screening. These fields can break selection rules and change values of matrix elements. Independent measurements of transmission performed on the same SI sample showed that FIR absorption in the region of small $B$ is negligible compared with the $1 s-2 p_{+}$peak. Therefore we conclude that the first matrix element in (1) in the SI material is not significantly different from the $n$-type. The strong increase of the photoconductive signal should come from an increase of the second term in Eq. (1). This probability is strongly influenced by the electric field $E$, i.e. the matrix element increases with increasing $E$.

The authors wish to express their gratitude to K. Kożuchowski for his help in transmission experiments.

\section{References}

[1] G.E. Stillman, C.M. Wolfe, J.O. Dimmock, in Semiconductors and Semimetals, Vol. 12, Eds. R.K. Willardson, A.C. Beer, Academic Press, New York 1977, p. 169. 
[2] Sh.M. Kogan, B.I. Sedunov, Sov. Phys.-Solid State 8, 1898 (1967).

[3] M.L. Sadowski, K. Karpierz, M. Grynberg, Phys. Rev. B 43, 7332 (1991).

[4] K. Karpierz, M.L. Sadowski, M.Grynberg, in Proc. 20th Int. Conf. Phys. Semicond., Thessaloniki 1990, Eds. E.M. Anastassakis, J.D. Joannopoulos, World Scientific, Singapore 1990, p. 609.

[5] D. Paget, P.B. Klein, Phys. Rev. B 34, 971 (1986).

[6]. Sh.M. Kogan, T.M. Lifshits, Phys. Status Solidi A 39, 11 (1977).

[7] J. Simola, J. Virtamo, J. Phys. B 11, 3309 (1978). 